\title{
Radiative corrections to neutrino-majoron couplings *
}

\author{
Juha T. Peltoniemi ${ }^{\dagger}$ \\ Research Institute for Theoretical Physics, Box 9, 00014 University of Helsinki, Finland.
}

(October 13, 2018)

\begin{abstract}
It is claimed that radiative corrections maintain the proportionality between the neutrino mass and the neutrino-majoron coupling and never give rise to enhanced decay rates in conventional majoron models. The coupling of a majoron to neutrinos is calculated at one loop level in various models, including the singlet majoron model and the Zee model with a majoron. When the respective corrections to the mass matrix are taken into account the wouldbe non-diagonal terms in the neutrino-majoron coupling are rotated away. It is pointed out that the coincidence between neutrino mass matrices and neutrino-majoron couplings is not accidental, but is a general consequence of Nöther's theorem. Nöther's theorem also implies that the majoron coupling to charged fermions is always diagonal in the fermion mass basis, and it vanishes completely in the singlet majoron models.
\end{abstract}

$12.15 \mathrm{Lk}, 12.60 \mathrm{Fr}, 13.15+\mathrm{g}, 13.35 \mathrm{Hb}, 14.60 \mathrm{Lm}, 14.80 \mathrm{Mz}$

Typeset using REVTEX

*hep-ph/9511416

HU-TFT-95-70

†Juha.Peltoniemi@Helsinki.fi 


\section{INTRODUCTION}

Most of the models explaining naturally a small neutrino mass break the global B-Lsymmetry that is present in the Standard Model. Here is considered the case that the symmetry is broken spontaneously, by a non-zero vacuum expectation value of a new scalar field. As a consequence of this, there will arise a massless Goldstone boson, majoron $(J)$.

In models with a massless majoron a massive neutrino may decay to a lighter neutrino state and a majoron. The life time of a neutrino is given by

$$
\tau_{\nu}=\frac{32 \pi}{|\xi|^{2} m_{\nu}}
$$

where $\xi$ is the non-diagonal majoron coupling, and $m_{\nu}$ is the mass of the decaying neutrino, and the daughter neutrino is assumed to be much lighter.

If the neutrinos are expected to form the dark matter, or part of it, they should be sufficiently long lived, i.e. should have a life time at least as long as the age of the universe $\left(t_{0} \sim 10^{17} \mathrm{~s}\right)$. On the other hand, a sufficiently rapid decay allows the neutrinos to have masses above $100 \mathrm{eV}$, forbidden for stable neutrinos. To be consistent with the present status of the universe, one requires [1, 2]

$$
\tau_{\nu}<t_{0} h^{4}\left(\frac{100 \mathrm{eV}}{m_{\nu}}\right)^{2},
$$

where $h$ is the normalized Hubble constant. The models of large scale structure formation impose limits that are several magnitudes more stringent, though more model dependent [3].

Typically the neutrino-majoron coupling is equal to the neutrino mass divided by the vacuum expectation value of the scalar field. Although this coupling itself may not be small, the decay amplitudes may be strongly suppressed. This is due to the fact that the majoron coupling matrix will be be diagonalised simultaneously with the mass matrix (just as the standard model higgs coupling is diagonal in the mass basis). For example, in the triplet majoron model the tree level majoron coupling is exactly diagonal in the mass basis.

In models where some components of the mass matrix are generated by a different mechanism than others, due to the different quantum numbers of the neutrinos involved, the coincidence of the mass and majoron coupling is broken. The simplest example is the see-saw model [4,5] with a singlet majoron [6], where only the Majorana mass of the righthanded sector breaks the lepton symmetry, and the Dirac mass components obey it. Thus non-diagonal majoron couplings arise at tree level, though these may be very small due to a characteristic hierarchy of these models [7].

It is not so evident that this structure is maintained beyond the tree level. Indeed, it has been claimed [8.9] that radiative corrections would induce non-diagonal components to the neutrino-majoron couplings. According to Ref. [8], the graph $7 \mathrm{~d}$ should allegedly induce a sufficiently large non-diagonal coupling to make light neutrinos essentially unstable in the triplet majoron model.

Here I will show that the appearance of non-diagonal elements is not true. Since we are actually interested in the form of the majoron coupling in the true mass basis, it is not enough 
to consider the corrections to the majoron coupling, but one should also take into account the respective corrections into the mass matrix. It will turn out that the mass eigenbasis is also rotated in such a way that the majoron coupling returns to the diagonal form. This is shown generally, in a model independent way, using the conservation of currents. To be sure, it is also proven explicitly in several models, by calculating the loops. Previously it has been shown that in a simplified singlet majoron model the radiative corrections do not enhance the decay [10].

In this paper I will calculate at one loop level the neutrino-majoron coupling in the singlet majoron model explicitly, more generally than in [10]. It is found that radiative corrections to the mass matrix coincide with the corrections to the majoron couplings, so that the neutrino decay is described sufficiently well by the tree level result. It is shown also that at one loop level the neutrino-majoron coupling is exactly diagonal (in the mass eigenbasis) in the majoronic version of the Zee model [11,12]. It is also argued that the results apply

to other similar models, like the triplet, doublet [13,14], singlet-triplet, doublet-triplet or singlet-doublet-triplet majoron models, as well as other radiative models. It is evident that this is true also for more complicated models where the majoron is incorporated in some Susy or GUT scenario whose low energy characteristics resemble the models considered here.

It has also been widely believed that radiative corrections would induce a coupling of a majoron to charged leptons, even when the tree level coupling would be zero. Because of these couplings the heavy leptons might then decay as $\ell_{2}^{-} \rightarrow \ell_{1}^{-} J$. However, the current argument implies that the radiative corrections do not induce any non-diagonal couplings, and in models where the majoron is a singlet the majoron does not couple at all to any charged fermion.

\section{GENERAL APPROACHES}

In Ref. 110 it was claimed that the diagonality of the majoron coupling is a direct consequence of the renormalisation procedure. According to this principle, any results obtained at the symmetric phase, i.e. for a zero vacuum expectation value, should be analytically continuable to the phase where this symmetry is hidden due to the arising non-zero vacuum expectation value. Hence the coincidence of mass and scalar couplings should be manifest. However, this kind of treatment is valid only for renormalisable dimension 4 terms. Higher dimension terms can arise already at one loop level.

Another approach based on Nöther's theorem was presented in Ref. [13], for the doublet majoron model. Below this is generalized to an arbitrary model.

Majoron models always contain two U(1) symmetries, one associated with the hypercharge $\mathrm{Y}$ and the other with the lepton number L. For each symmetry one can define a current

$$
\begin{aligned}
& J_{\mu}^{L}=\sum_{h} L_{h} \Phi_{h}^{\dagger} \partial_{\mu} \Phi_{h}+\sum_{f} L_{f} \bar{\Psi}_{f} \gamma_{\mu} \Psi_{f}, \\
& J_{\mu}^{Y}=\sum_{h} Y_{h} \Phi_{h}^{\dagger} \partial_{\mu} \Phi_{h}+\sum_{f} Y_{f} \bar{\Psi}_{f} \gamma_{\mu} \Psi_{f},
\end{aligned}
$$

where $h$ runs over scalar states and $f$ over fermion states, and $L_{i}$ and $Y_{i}$ label the lepton 
number and the hypercharge of particle $i$, respectively. Whenever the symmetry is exact these currents are conserved, i.e.

$$
\partial^{\mu} J_{\mu}^{L, Y}=0
$$

Furthermore, the currents are conserved also after the spontaneous breaking of the symmetry, as long as the underlying theory itself is renormalisable and anomaly free. However, anomalies break Nöther's theorem by instanton effects, and for exactly this reason we should expand the lepton number symmetry to include baryons $(B-L)$.

In the phase of spontaneously broken symmetries we can shift the neutral components by their vacuum expectation values, so that the equations (3) and (耳) can be written as

$$
\begin{aligned}
& J_{\mu}^{L}=\sum_{h} L_{h}\left\langle\Phi_{h}\right\rangle \partial_{\mu} \operatorname{Im}\left\{\Phi_{h}\right\}+j_{\mu}^{L} \\
& J_{\mu}^{Y}=\sum_{h} Y_{h}\left\langle\Phi_{h}\right\rangle \partial_{\mu} \operatorname{Im}\left\{\Phi_{h}\right\}+j_{\mu}^{Y},
\end{aligned}
$$

where the $j_{\mu}$ include the other terms of Eqs. (3) and (41). The first order terms in (7) consist of the unphysical Goldstone mode associated with the $Z$ boson, by definition, while those in (6) include both the unphysical higgs and the true Goldstone boson, majoron. The majoron is defined as

$$
J=C \sqrt{2} \sum_{h}\left(\sum_{i} Y_{i}^{2} v_{i}^{2} L_{h}-\sum_{i} L_{i} Y_{i} v_{i}^{2} Y_{h}\right) v_{h} \operatorname{Im}\left\{\Phi_{h}\right\},
$$

where

$$
C^{-1}=\sqrt{\sum_{h}\left(\sum_{i} Y_{i}^{2} v_{i}^{2} L_{h}-\sum_{i} L_{i} Y_{i} v_{i}^{2} Y_{h}\right)^{2} v_{h}^{2}} .
$$

Arranging the terms, using the conservation of currents (5), and eliminating the would-be Goldstone mode of $Z$, one obtains

$$
\partial^{\mu} \partial_{\mu} J+2 C \partial^{\mu}\left(\sum_{i} Y_{i}^{2} v_{i}^{2} j_{\mu}^{L}-\sum_{i} L_{i} Y_{i} v_{i}^{2} j_{\mu}^{Y}\right)=0
$$

Now one can see that the neutrino-majoron coupling should be of the form

$$
\begin{aligned}
\mathcal{L}_{J}= & C\left[\left(\sum_{h} Y_{h}^{2} v_{h}^{2}\right)\left(\sum_{f} U_{a f}^{\dagger} L_{f} U_{f b}\right)\right. \\
& \left.-\left(\sum_{h} L_{h} Y_{h} v_{h}^{2}\right)\left(\sum_{f} U_{a f}^{\dagger} Y_{f} U_{f b}\right)\right] J \bar{\nu}_{a}\left(\overleftarrow{\partial}_{\mu}-\vec{\partial}_{\mu}\right) \gamma^{\mu} \nu_{b} .
\end{aligned}
$$

Using the Dirac equation (for Majorana neutrinos) one can extract the coupling constant

$$
\begin{aligned}
\xi_{a b}= & C\left(\sum_{h} Y_{h}^{2} v_{h}^{2}\right)\left(\sum_{f} U_{a f}^{\dagger} L_{f} U_{f b}\right)\left(m_{a}+m_{b}\right) \\
& -C\left(\sum_{h} L_{h} Y_{h} v_{h}^{2}\right)\left(\sum_{f} U_{a f}^{\dagger} Y_{f} U_{f b}\right)\left(m_{a}+m_{b}\right),
\end{aligned}
$$


where $m_{a, b}$ are Majorana masses of neutrinos a,b. (For Dirac neutrinos the result is different.) One sees immediately that a necessary and sufficient condition for the majoron coupling being diagonal in the neutrino mass basis is that all neutrinos are identical in their hypercharge and lepton numbers

To be sure that the above reasoning is correct, below it will be proven explicitly that one loop corrections give results in full agreement with the above results, in the singlet majoron model with the see-saw mechanism and the Zee model.

\section{SINGLET MAJORON MODEL}

\section{A. Tree level}

The singlet majoron model [15] is the most evident extension to the standard model containing a majoron. This model involves, beside the Standard Model particles, three right handed neutrinos, with a canonical lepton number 1, and a neutral scalar $\sigma$ with lepton number 2. The Yukawa interactions of neutrinos are given by

$$
\Phi^{0} \bar{\nu}_{L} f \nu_{R}+\sigma \bar{\nu}_{R} h \nu_{R}
$$

where $\Phi$ is the standard model higgs doublet, and $\nu_{L}$ and $\nu_{R}$ are 3 dimensional vectors representing the three neutrino flavors, and the coupling constants $f$ and $h$ are respectively $3 \times 3$ matrices.

After the spontaneous symmetry breaking by a non-zero vacuum expectation value of $\sigma$,

$$
\langle\sigma\rangle \equiv \frac{w}{\sqrt{2}}
$$

there will be a massless majoron, $J$, as the imaginary part of the field,

$$
\sigma=\frac{1}{\sqrt{2}}(w+\rho+i J) .
$$

The standard model higgs can also be decomposed as

$$
\Phi^{0}=\frac{1}{\sqrt{2}}\left(v+H^{0}+i \phi^{0}\right)
$$

with $\left\langle\Phi^{0}\right\rangle=v / \sqrt{2}$. A cross coupling of the scalars will induce a mixing between the real parts. Define the mass eigenstates as

$$
\begin{aligned}
& H_{1}=\cos \theta H^{0}-\sin \theta \rho, \\
& H_{2}=\sin \theta H^{0}+\cos \theta \rho,
\end{aligned}
$$

with masses $M_{1}$ and $M_{2}$. The Goldstone modes do not mix, as $\sigma$ carries no hypercharge, and $\Phi$ has no lepton number.

The tree level neutrino mass matrix reads now 


$$
\mathcal{M}_{\circ}=\frac{1}{\sqrt{2}}\left[\begin{array}{cc}
0 & f v \\
f^{\dagger} v & h w
\end{array}\right] \equiv\left[\begin{array}{cc}
0 & M_{D} \\
M_{D}^{\dagger} & M_{M}
\end{array}\right]
$$

where $M_{D}$ and $M_{M}$ are $3 \times 3$ matrices. This can be block diagonalised by the matrix [16, 17]

$$
U=\left[\begin{array}{cc}
1-\frac{1}{2} \epsilon \epsilon^{\dagger} & \epsilon-\frac{3}{2} \epsilon \epsilon^{\dagger} \epsilon \\
\epsilon^{\dagger}+\frac{3}{2} \epsilon^{\dagger} \epsilon \epsilon^{\dagger} & 1-\frac{1}{2} \epsilon^{\dagger} \epsilon
\end{array}\right]
$$

to the order of $\epsilon^{4}$, where

$$
\epsilon=M_{D} M_{M}^{-1}
$$

After the rotation we obtain the mass matrix

$$
\mathcal{M}_{\circ}=\left[\begin{array}{cc}
\widehat{m} & 0 \\
0 & \breve{m}
\end{array}\right]+\left[\begin{array}{ll}
O\left(\epsilon^{6}\right) & O\left(\epsilon^{5}\right) \\
O\left(\epsilon^{5}\right) & O\left(\epsilon^{4}\right)
\end{array}\right]
$$

where

$$
\begin{aligned}
& \widehat{m}=-\epsilon M_{M} \epsilon^{\dagger}+\frac{1}{2}\left(\epsilon M_{M} \epsilon^{\dagger} \epsilon \epsilon^{\dagger}+\epsilon \epsilon^{\dagger} \epsilon M_{M} \epsilon^{\dagger}\right), \\
& \breve{m}=M_{M}+\frac{1}{2}\left(M_{M} \epsilon^{\dagger} \epsilon+\epsilon^{\dagger} \epsilon M_{M}\right) .
\end{aligned}
$$

The remaining submatrix of the light neutrinos can be diagonalised by a further rotation in that subpart by a $(3 \times 3)$ unitary matrix.

Define now the majoron coupling as

$$
-i \xi_{i j} J \bar{\nu}_{i}^{c} \gamma_{5} \nu_{j}
$$

One sees that at the tree level we have $\xi=h / \sqrt{2}=M_{M} / w$, and in the weak basis only the $3 \times 3$ submatrix of the right-handed states in $\xi$ is non-zero. After the block diagonalisation one has

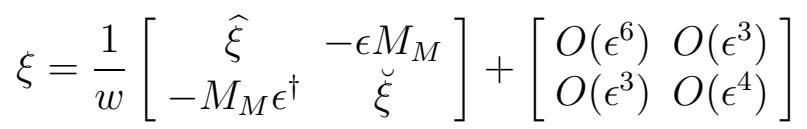

where

$$
\begin{aligned}
& \widehat{\xi}=\epsilon M_{M} \epsilon^{\dagger}-\frac{3}{2}\left(\epsilon M_{M} \epsilon^{\dagger} \epsilon \epsilon^{\dagger}+\epsilon \epsilon^{\dagger} \epsilon M_{M} \epsilon^{\dagger}\right) \\
& \breve{\xi}=M_{M}-\frac{1}{2}\left(M_{M} \epsilon^{\dagger} \epsilon+\epsilon^{\dagger} \epsilon M_{M}\right)
\end{aligned}
$$

The elements for the coupling between the heavy and the light mass eigenstates are substantial, but the couplings of both the heavy and the light mass eigenstates are in themselves separately diagonal in the mass basis, at $O\left(\epsilon^{3}\right)$. As a result of this, the decay of a light massive neutrino to a lighter neutrino and a majoron is suppressed by $\epsilon^{4}$ at the tree level. The leading order for neutrino decay is now $\epsilon^{4}$, the respective couplings can be written as 16

$$
\widehat{\xi}^{\prime}=-\frac{1}{w}\left(\widehat{m} \epsilon \epsilon^{\dagger}+\epsilon \epsilon^{\dagger} \widehat{m}\right) .
$$

For all plausible values of the parameters, this is too small to induce a rapid neutrino decay. 


\section{B. Radiative corrections}

Calculate now exactly the most important radiative corrections to the mass and majoron coupling. The graphs contributing to the mass are presented in figs. 3 and 5 while the graphs with an external majoron are depicted in figs. 2, 4 and 6. To obtain maximum clarity, we do our calculations in the weak (chirality) basis of the external states, labeling the lefthanded states with $i, j$ and the right-handed states by $a, b$. The readers interested in a more elaborate treatment in the tree level mass basis with further rerotations into the corrected mass basis should consult the ref. [10]. The internal particles are always considered to be mass eigenstates. Moreover, at the desired accuracy we can assume that the heavy mass eigenstates coincide with the right-handed states. Hence the $\sigma$ coupling can be taken to be diagonal. All the loop calculations are performed in the Feynman-t'Hooft gauge.

Let us start with the corrections to the left-handed states. The graphs contributing to the mass matrix are presented in figure 1. The two graphs containing scalars diverge separately, but their sum is finite, giving

$$
\begin{aligned}
\delta m_{i j}^{\left(H_{i}, \phi\right)}= & \frac{1}{32 \pi^{2}} \sum_{a} f_{i a} \check{m}_{a} f_{j a} \\
& {\left[\left(M_{1}^{2}-M_{Z}^{2}\right) I\left(\check{m}_{a}, M_{1}, M_{Z}\right)\right.} \\
& \left.-\sin ^{2} \theta\left(M_{2}^{2}-M_{1}^{2}\right) I\left(\check{m}_{a}, M_{1}, M_{2}\right)\right],
\end{aligned}
$$

where

$$
\begin{aligned}
& I\left(m_{1}, m_{2}, m_{3}\right)= \\
& \frac{m_{1}^{2} m_{2}^{2} \ln \frac{m_{1}^{2}}{m_{2}^{2}}+m_{2}^{2} m_{3}^{2} \ln \frac{m_{2}^{2}}{m_{3}^{2}}+m_{3}^{2} m_{1}^{2} \ln \frac{m_{3}^{2}}{m_{1}^{2}}}{\left(m_{2}^{2}-m_{1}^{2}\right)\left(m_{3}^{2}-m_{2}^{2}\right)\left(m_{1}^{2}-m_{3}^{2}\right)},
\end{aligned}
$$

and $\theta$ is the scalar mixing angle. Respectively, the scalar graphs in figure refkuvaLLJ yield the majoron coupling

$$
\begin{aligned}
\delta \xi_{i j}^{\left(H_{i}, \phi\right)}= & \frac{1}{32 \sqrt{2} \pi^{2}} \sum_{a} f_{i a} h_{a} f_{j a} \\
& {\left[\left(M_{1}^{2}-M_{Z}^{2}\right) I\left(\check{m}_{a}, M_{1}, M_{Z}\right)\right.} \\
& \left.-\sin ^{2} \theta\left(M_{2}^{2}-M_{1}^{2}\right) I\left(\check{m}_{a}, M_{1}, M_{2}\right)\right]
\end{aligned}
$$

The graph with $Z$ exchange (11 b) requires a more careful treatment. It is now compulsory to take all the neutrino states into account as the internal lines: ignoring the light states

would leave a diverging integral. From the definition of $\epsilon$ one sees that these two sets of graphs are indeed of the same order in $\epsilon$, and can be summed. In the lowest order of $\epsilon$ one obtains

$$
\delta m_{i j}^{(Z)}=\frac{g^{2}}{256 \pi^{2} \cos ^{2} \theta_{W}} \sum_{a} \epsilon_{i a} \check{m}_{a}^{3} I\left(\check{m}_{a}, M_{Z}, 0\right) \epsilon_{a j}^{\dagger}
$$


The $Z$ contribution to the majoron coupling (Fig. 2 b) is respectively

$$
\begin{aligned}
\delta \xi_{i j}^{(Z)}= & \frac{g^{2}}{256 \sqrt{2} \pi^{2} \cos ^{2} \theta_{W}} \\
& \sum_{a} \epsilon_{i a} \check{m}_{a}^{2} h_{a} I\left(\check{m}_{a}, M_{Z}, 0\right) \epsilon_{a j}^{\dagger} .
\end{aligned}
$$

where the equation (23) was used.

Consider then the left-right components. The Dirac mass term is corrected by the graph 3. We obtain

$$
\begin{aligned}
\delta m_{i a}= & \sum_{b j} \frac{1}{32 \pi^{2}} f_{i b} \check{m}_{b} \epsilon_{b j}^{\dagger} f_{j a} \\
& {\left[\cos ^{2} \theta I\left(\check{m}_{b}, M_{1}, M_{Z}\right)+\sin ^{2} \theta I\left(\check{m}_{b}, M_{2}, M_{Z}\right)\right] . }
\end{aligned}
$$

At tree level there was no coupling of a majoron between a left- and a right-handed neutrino. At one loop level, the graphs presented in figures 4 would contribute to such a coupling. However, it turns out that these graphs cancel so that there is no respective majoron coupling at all. This is shown in the Appendix.

Continue then with the components involving only the right-handed neutrino states. The one-loop correction to the mass term, from figure 5, can be expressed as

$$
\begin{aligned}
\delta m_{a a}= & \frac{1}{32 \pi^{2}} h_{a}^{2} \check{m}_{a}\left[\cos ^{2} \theta M_{2}^{2} I\left(\check{m}_{a}, M_{2}, 0\right)\right. \\
& \left.+\sin ^{2} \theta M_{1}^{2} I\left(\check{m}_{a}, M_{1}, 0\right)\right] .
\end{aligned}
$$

Other contributions appear at higher order of $\epsilon$, being thus ignorable.

There are now four graphs contributing to the majoron coupling. The graph (6a), with the majoron coupling to the scalar vertex gives

$$
\begin{aligned}
\delta \xi_{a}^{(\rho J)}= & \frac{1}{32 \pi^{2}} h_{a}^{2} \frac{\check{m}_{a}}{w}\left[M_{2}^{2} \cos ^{2} \theta I\left(\check{m}_{a}, M_{2}, 0\right)\right. \\
& \left.+M_{1}^{2} \sin ^{2} \theta I\left(\check{m}_{a}, M_{1}, 0\right)\right] .
\end{aligned}
$$

There is another graph with $\rho$ and $J$ lines interchanged which gives exactly the same contribution. The graphs (6) b) with the majoron coupling to the fermion line diverge separately, but together they sum up to the finite result

$$
\begin{aligned}
\delta \xi_{a}^{(\nu)}=- & \frac{1}{32 \sqrt{2} \pi^{2}} h_{a}^{3}\left[\cos ^{2} \theta M_{2}^{2} I\left(\check{m}_{a}, M_{2}, 0\right)\right. \\
& \left.+\sin ^{2} \theta M_{1}^{2} I\left(\check{m}_{a}, M_{1}, 0\right)\right] .
\end{aligned}
$$

The rest of the contributing graphs can be ignored since they are of higher order in $\epsilon$.

Summing up all contributions, one sees that the one loop contributions to the majoron couplings are given by

$$
\delta \xi=\frac{1}{w}\left[\begin{array}{cc}
\delta m_{L} & 0 \\
0 & \delta m_{R}
\end{array}\right] .
$$


From this it follows that after diagonalising the mass matrix, by a redefined rotation matrix, the resulting majoron coupling is still diagonal in the lowest order, and the non-diagonal majoron couplings are obtained from the same equation (29) as in the tree level case (with only the quantities $m$ and $\epsilon$ involving the tiny loop corrections).

\section{RADIATIVE MODELS}

\section{A. Majoronic Zee model}

The model of Zee [11,12] is the simplest mechanism to generate the neutrino masses radiatively. This model is often incorporated with a global symmetry, which can be a flavor dependent Zeldovich-Konopinski-Mahmoud symmetry [18,19] or a modification of it [20,21]. Here I restrict on the variant where this symmetry is the canonical lepton number symmetry, whose spontaneous breakdown then produces the majoron.

There are several ways to insert the majoron to the Zee model. The simplest is to assume the majoron to be composed of the doublets present in the Zee model [13]. This variant is, however, incompatible with the LEP results, so one has to add more higgses. In the following is considered the case that the majoron emerges from a singlet field. Another possibility

would be to add a third doublet, in the same spirit as in the model of Ref. [14. The final results are equal for all cases, up to a trivial scalar mixing factor in the majoron couplings.

The considered model has the minimal Standard Model fermion sector, including no right-handed neutrinos, with canonical lepton numbers. The higgs sector consists of the standard higgs doublet $\Phi$, one singly charged singlet scalar $\eta^{-}$, one additional doublet $\varphi$, and a neutral scalar $\sigma$. Both of the singlets have lepton number 2 while the doublets do not carry any lepton number.

Only one of the doublets couples to leptons, and the Yukawa couplings to neutrinos are given by

$$
h_{i} \Phi^{+} \bar{\nu}_{L i} l_{R i}^{-}+f_{i j} \eta^{-} \bar{l}_{L i}^{-} \nu_{L j}
$$

where $f_{i j}$ is antisymmetric. The $\sigma$ field couples only to the scalars, with a quartic coupling

$$
\lambda \Phi^{T} \varphi \eta^{-} \sigma
$$

where $\lambda$ is a dimensionless coupling constant.

After the spontaneous symmetry breakings we have 3 charged scalars, one of which is eaten up by $W$. It is sufficient to describe their mixing by two mixing angles: $\alpha$ is the mixing between physical and unphysical components,

$$
\tan \alpha=\frac{\langle\Phi\rangle}{\langle\varphi\rangle}
$$

and $\beta$ is the mixing between the physical eigenstates,

$$
\tan 2 \beta=\frac{4 \sqrt{2} \lambda\langle\sigma\rangle}{g} \frac{M_{W}}{M_{\phi}^{2}-M_{\eta}^{2}},
$$


where $M_{\phi}$ and $M_{\eta}$ are the diagonal mass terms in the weak basis. One sees easily that the coupling of the majoron to the physical charged bosons is purely non-diagonal in the mass basis, the respective coupling constant being $i \lambda \sqrt{\langle\Phi\rangle^{2}+\langle\varphi\rangle^{2}} / \sqrt{2}$.

The mass is given by the graph (a) of the figure 9 [20]

$$
\begin{aligned}
m_{i k}= & \frac{f_{i k} g \sin 2 \beta \cot \alpha}{32 \pi^{2} M_{W}}\left(M_{2}^{2}-M_{1}^{2}\right) \\
& {\left[I\left(m_{i}, M_{1}, M_{2}\right)-I\left(m_{k}, M_{1}, M_{2}\right)\right], }
\end{aligned}
$$

where the function $I$ is the same as before (31), and $M_{1}$ and $M_{2}$ are the mass eigenstates. In the limit $m_{k} \ll M_{j}$ the mass reads

$$
m_{i k}=\frac{f_{i k} g \sin 2 \beta \cot \alpha}{32 \pi^{2} M_{W}}\left(m_{i}^{2}-m_{k}^{2}\right) \ln \frac{M_{1}^{2}}{M_{2}^{2}} .
$$

This limit is sufficient for most practical applications.

The majoron coupling (Figure 9 b) is respectively given by

$$
\begin{aligned}
\xi_{i k}= & \frac{f_{i k} g \lambda \cot \alpha \sqrt{\langle\Phi\rangle^{2}+\langle\varphi\rangle^{2}}}{16 \sqrt{2} \pi^{2} M_{W}} \\
& {\left[I\left(m_{i}, M_{1}, M_{2}\right)-I\left(m_{k}, M_{1}, M_{2}\right)\right] . }
\end{aligned}
$$

Using the definition of the mixing angle and taking the heavy scalar limit one obtains

$$
\xi_{i k}=\frac{f_{i k} g \sin 2 \beta \cot \alpha}{32 \pi^{2} w M_{W}}\left(m_{i}^{2}-m_{k}^{2}\right) \ln \frac{M_{1}^{2}}{M_{2}^{2}} .
$$

Hence one has also in this model exactly

$$
m_{i j}=\xi_{i j} w
$$

at one loop level.

\section{B. Other radiative models}

It is obvious now that these results can be generalized to other radiative models with similar kind of loops. One example of them is the model considered in [22]. In it it was proposed that singlet neutrinos could be light, with radiatively generated masses. It can be shown that in that model the radiatively induced neutrino-majoron coupling is diagonal, and the neutrinos are essentially stable, justifying the motivation for the model of having the singlet neutrinos as dark matter.

A more trivial case is the model of Babu [23] with a singlet majoron. In that model the mass is generated at two loop level, and the majoron couples only to the charged scalar bosons. Hence it is free from any serious constraints from lepton phenomenology. The mass in now given by the graph 10a, while the majoron coupling results from 10]b. These graphs are trivially proportional to each others.

There is a plethora of other models with radiative generation of masses. Many of them use non-canonical family dependent lepton number assignment or light right-handed neutrinos. In such scenarios the neutrino decay can take place via the lowest order graphs, and it may not be suppressed essentially. 


\section{TRIPLET MAJORON MODEL}

The triplet majoron model [24] is the simplest alternative to produce neutrino masses without right-handed neutrinos. It was formerly very popular, but the LEP results were rather detrimental to this model, excluding it in its simplest forms. The triplet scenario itself is not dead, however, it survives as a part of more complicated models, e.g. the singlettriplet model. Hence it is well motivated to study the triplet model as a starting point to more elaborate models.

In the triplet majoron models one adds to the minimal standard model a triplet field $\Delta$, with hypercharge 2 and lepton number 2 . It couples to lepton $\operatorname{doublets}\left(\Psi_{L}\right)$ via the coupling

$$
\zeta_{i j} \Psi_{L i}^{T} \Delta \Psi_{L j}
$$

The lepton number is now broken spontaneously by the vacuum expectation value of $\Delta$, denoted by $\langle\Delta\rangle \equiv u / \sqrt{2}$. Even though there are no cross couplings between the scalars, the gauge interactions mix the Goldstone modes, and the majoron is a linear combination of the imaginary parts of both neutral higgses,

$$
J=\sqrt{2} \operatorname{Im}\left\{\frac{2 u \Phi^{\circ}-v \Delta^{\circ}}{\sqrt{v^{2}+4 u^{2}}}\right\} .
$$

The unphysical higgs field is then orthogonal to the majoron.

The neutrino-majoron coupling is now at tree level

$$
\xi_{0}=\frac{m_{0}}{u} \frac{v}{\sqrt{v^{2}+4 u^{2}}} .
$$

The majoron has also a coupling to the charged fermions, via the doublet component.

In Ref. [8] it was claimed that the graph 7a would yield the non-diagonal couplings. Indeed, it contributes to the majoron couplings, rotating the neutrino-majoron coupling matrix from its original basis. However, there are other graphs to be taken into account, for instance the graph $7 \mathrm{~b}$ that contributes to the mass matrix. One must be very careful with the graphs in this case, due to the peculiarities of this case. For example, the graph (a) gives in fact a higher dimension term $\nu \nu \Delta \Phi \Phi$, so that the majoron coupling emerges only after $\Phi$ gets a vacuum expectation value, while the respective mass correction (b) is there even if $\Phi$ would have zero vacuum expectation value. Furthermore, the mass correction is divergent so that an appropriate renormalisation procedure should be applied for the results to make sense. The renormalisation then can and should be done in the symmetric phase, so that the results remain to be valid also after the breaking of the symmetries, for the shifted fields.

Being now convinced that the argument of current conservation is sufficient, the calculation of the loops is skipped. Instead the results obtained in the section II by Nöther's theorem are applied. From the formula (12) one obtains directly

$$
\xi=m \frac{v}{u \sqrt{v^{2}+4 u^{2}}},
$$

valid after infinite radiative corrections. Hence the majoron coupling remains strictly diagonal, contradictory to the claims of ref. [8]. 


\section{OTHER MODELS}

\section{A. Singlet-triplet model}

The simplest cure to the triplet majoron model is introducing a singlet higgs. In the singlet-triplet majoron model the majoron is given by the composition [7]

$$
J=\sqrt{2} \operatorname{Im}\left[\frac{-2 v u^{2} \Phi^{o}+w\left(v^{2}+4 u^{2}\right) \sigma+u v^{2} \Delta^{o}}{\sqrt{4 v^{2} u^{2}+w^{2}\left(v^{2}+4 u^{2}\right)+u^{2} v^{4}}}\right] .
$$

One can choose the parameters so that the majoron is predominantly a singlet, thus avoiding the LEP constraint. The new singlet couples only to the other scalars, via the coupling

$$
\lambda^{\prime} \sigma \Phi^{T} \Delta \Phi
$$

There will be no new graphs contributing to the mass or to the majoron coupling of neutrinos, hence the above results apply, corrected only by some mixing factors. Thus the neutrinos can be considered absolutely stable in the singlet-triplet majoron model with the minimum fermion contents.

Allowing the existence of the right-handed neutrinos would lead to a case where the neutrino mass is generated mixedly by the triplet field and by the singlet field through the see-saw mechanism. It is reminded that despite the two different mechanisms for the light neutrino mass, the neutrino-majoron coupling remains diagonal at $\epsilon^{2}$ [7]. At one loop level the result is corrected by all the graphs considered for both the singlet and the triplet majoron model, with only the scalar mixing factors being more complicated. There appear also some new graphs due to the mixing of the majoron with the doublet higgs. Again, instead of calculating the graphs, the result (12) is applied, giving

$$
\xi=\frac{v^{2} m+\left(v^{2}+2 u^{2}\right)\left(m \epsilon \epsilon^{\dagger}+\epsilon \epsilon^{\dagger} m\right)}{\sqrt{4 v^{2} u^{2}+w^{2}\left(v^{2}+4 u^{2}\right)+u^{2} v^{4}}},
$$

where the non-diagonal parts emerge from the latter terms.

\section{B. Doublet majoron models}

The doublet majoron models 13 have also suffered from the results of LEP. In the simplest version of it, one adds to the standard model a new scalar doublet with lepton number 1 . Then we need also three right-handed neutrinos which carry no lepton number, so that their Majorana mass term can appear in the bare Lagrangian. As a result of the spontaneous breaking of the lepton number (and $S U(2) \times U(1)$ as well) the neutrinos get Dirac mass terms. The mass matrix is diagonalised exactly as in the singlet majoron case, and the light neutrinos get their masses due to the see-saw mechanism.

Apart from the singlet majoron model, the majoron couples only between the left- and right-handed neutrinos. Due to the explicit non-coincidence of the mass matrix and majoron 
couplings, the majoron coupling can be non-diagonal even at the tree level. In the mass basis the majoron coupling is given by

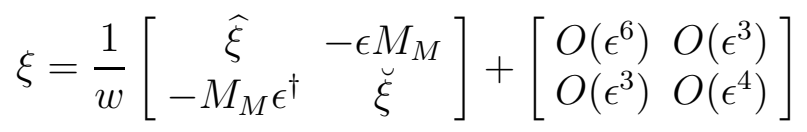

where

$$
\begin{aligned}
& \widehat{\xi}=-2 \epsilon M_{M} \epsilon^{\dagger}+2 \epsilon M_{M} \epsilon^{\dagger} \epsilon \epsilon^{\dagger}+2 \epsilon \epsilon^{\dagger} \epsilon M_{M} \epsilon^{\dagger} \\
& \breve{\xi}=2 M_{M}-2 M_{M} \epsilon^{\dagger} \epsilon-2 \epsilon^{\dagger} \epsilon M_{M}
\end{aligned}
$$

Coincidentally, the non-diagonal majoron coupling of the light neutrinos is given by the same equation as that of the singlet majoron model (with an insignificant sign difference). However, in the doublet majoron model (despite the apparently similar formula) the neutrino majoron coupling constant is generally larger, since the scale $w$ is supposed to be lower than in the singlet majoron model.

It is easy to verify explicitly that the result is valid also with the radiative corrections. All the relevant loops are well-behaving graphs, structurally similar to those considered in singlet majoron case, giving together convergent results. Omitting the explicit proof, one concludes that the loops do not add any relevant contribution, as follows from the current conservation.

Recently a variant of the doublet majoron model [14] was presented that adds another (third) doublet to the model. This allows the $\rho$ field to be heavy enough to disallow the decay $Z \rightarrow J \rho$, without invoking a too big majoron coupling to the charged leptons. Another remedy is to add a singlet scalar. In neither case there will arise any new contributions up to one loop level, since the introduced particles do not couple to leptons.

\section{Doublet-triplet models}

For completeness, consider finally the theoretical doublet-triplet model. In this scenario the majoron consists of a triplet and a doublet that does not have to be the standard model doublet. Since the new doublet does not couple to leptons, the results of the triplet majoron models apply, with some trivial corrections due to mixing. Hence, the majoron is now given by

$$
J=\sqrt{2} \operatorname{Im}\left[\frac{\left(w^{2}+4 u^{2}\right) \Phi^{\circ}-v w \varphi^{\circ}-2 u v \Delta^{0}}{\sqrt{\left(v^{2}+w^{2}+4 u^{2}\right)\left(w^{2}+4 u^{2}\right)}}\right],
$$

where $\Phi$ denotes the standard scalar doublet, $\varphi$ the new doublet and $\Delta$ the scalar triplet, and $v, w$ and $u$ are their vacuum expectation values, respectively. Its coupling to neutrinos reads

$$
\xi=m \frac{2 v}{\sqrt{\left(w^{2}+4 u^{2}\right)\left(v^{2}+w^{2}+4 u^{2}\right)}},
$$

being truly diagonal. 
With this particle contents one can avoid the inconsistency between $Z$ decay width and $\rho$ parameter, but the model yet fails to satisfy the astrophysical constraints for any natural choice of parameters. To cure that, one should add more higgses, and as long as they do not couple to leptons, there are no new one-loop graphs to be taken into account. In any case the general result guarantees that there are no new effects due to the higher order corrections.

\section{MAJORON COUPLING TO CHARGED FERMIONS}

It has been often speculated (see [16,25 for a few examples) that a substantial coupling between majorons and charged leptons would arise by radiative corrections. Since the phenomenology of the charged leptons is relatively well understood, the laboratory experiments, as well as astrophysical considerations, constrain such couplings severely.

We can derive the general majoron coupling to Dirac fermions using the same method of current conservation as for neutrinos. Hence we obtain

$$
\xi_{f}=-C\left(\sum_{h} L_{h} Y_{h} v_{h}^{2}\right) m_{f},
$$

for a fermion $\mathrm{f}$ with the standard quantum number assignments, and a Dirac mass $m_{f}$.

The above result (61) would imply that in the singlet majoron models the majoron does not couple to any other known fermion than neutrinos. Such a conclusion might also appear obvious from the initial form of the effective coupling, $\sigma^{\dagger} \sigma \bar{f} f$. In models where the majoron is composed of non-singlet components it couples to fermions already at the tree level, via its doublet component. However, according to (61), the majoron coupling should be always diagonal in all models with the standard fermion sector, and hence the decay $f \rightarrow f^{\prime} J$ should be forbidden.

Let us now consider as an example a specific model in more detail. The simplest case is a model with a singlet majoron that does not couple directly to fermions, but has a coupling with other scalars, introduced e.g. to generate radiative mass terms. Hence we assume three new scalars, $\chi_{1}, \chi_{2}$ and $\sigma$, where $\chi$ can be doubly charged scalars, as in [25], or some other representation. Now the loops depicted in the figure 11 would apparently induce a coupling of the majoron to the charged leptons. However, the coupling $J \chi_{i} \chi_{j}$ is antisymmetric, from which it directly follows that the graphs with different scalar mass eigenstates cancel. Hence the majoron coupling to the charged lepton vanishes in this model.

\section{CONCLUSIONS}

It was claimed that in models with spontaneously broken lepton number symmetries the radiative corrections do not enhance the invisible decay rates of neutrinos. The graphs with external majorons are always accompanied with similar graphs without majorons that contribute to the mass, and the resulting mass and majoron coupling matrices turn out to be simultaneously diagonal.

It was explicitly demonstrated that in the singlet majoron model the dominant component to neutrino decay appears at tree level, and the radiative corrections do not enhance it. 
It was found that the coincidence between the neutrino mass and the neutrino-majoron coupling is maintained also in models where both are generated radiatively. The neutrinomajoron coupling at one loop level was calculated exactly in the Zee model with a singlet majoron. It is evident that the result applies to other radiative models with similar ingredients.

A general proof for the coincidence between mass matrix and neutrino majoron coupling was given using Nöther's theorem. This result is valid for all well-behaving theories up to infinite radiative corrections.

We can conclude that the neutrino-majoron coupling is strictly diagonal, and the neutrinos are stable against a decay to a majoron in models where all neutrinos have identical lepton number and hypercharge. This is the case in all models with the minimal fermion content and a canonical lepton number assignment. Examples include triplet and doublet majoron models and their derivatives not containing the right-handed neutrinos.

The general arguments apply also to the coupling of majorons to other fermions. Hence in models where the majoron has a doublet component it couples to charged fermions at tree level, and the radiative corrections do not induce any non-diagonal components. In models where the majoron is made of singlets it does not couple at all to charged leptons or quarks having conventional quantum numbers. It was shown explicitly that in a specific singlet majoron model the one-loops apparently contributing to such a coupling vanish. These results do not necessarily apply for models with non-canonical lepton number assignments and exotic fermions.

The above arguments do not forbid higher dimension terms involving many majorons, like $\bar{f} f J J$, that could be induced radiatively. Nevertheless even though such terms would appear, they would be very small, especially the non-diagonal components. Moreover, the decay $f_{2} \rightarrow f_{1} J^{n}$ would be suppressed by phase space arguments.

It has been speculated that the global symmetry may be broken both explicitly and spontaneously [26]. This might destabilize neutrinos even in models where they are otherwise stable, if the majoron remains sufficiently massless. For instance, in the triplet majoron model, a small gravitationally induced neutrino mass of $\mathrm{O}\left(10^{-5}\right) \mathrm{eV}$ is sufficiently big to cause any neutrino considered relevant for the dark matter to decay in time scales much less than the age of the universe. On the other hand, in typical singlet majoron models the majoron coupling is so small that the gravitationally induced mass is not sufficient to enhance the decay rate.

The explicit symmetry breaking may also induce a mass to the majoron. Its mass could be as high as tens of $\mathrm{keV}$ in which case one does not talk about the decay of light neutrinos, even if the explicit symmetry breaking terms might induce sizable non-diagonal couplings. Many astrophysical constraints do not apply for such majorons which may give more freedom to models.

\section{ACKNOWLEDGEMENT}

This work was supported by the Spanish Ministry for Education and Science, and by Istituto Nazionale di Fisica Nucleare. I have benefitted from the discussions with Kimmo Kainulainen, Palash Pal and José Valle. 


\section{APPENDIX A: THE ABSENCE OF THE $\nu_{L} \nu_{R} J$ - COUPLING IN THE SINGLET MAJORON MODEL}

There was no majoron coupling between the left and right handed components at the tree level; neither will it arise by loops which is now shown explicitly, calculating the graphs of figure 4 . Summing all the fermionic contributions together, we obtain, for the $\phi$ exchange graph

$$
\begin{aligned}
-i \gamma_{5} \delta \xi_{a i}^{(\phi)}= & \sum_{b j} i \int \frac{f_{a j} \gamma_{5}}{\sqrt{2}} \epsilon_{j b} i \frac{\not+\check{m}_{b}}{k^{2}-\check{m}_{b}^{2}} \frac{-h_{b} \gamma_{5}}{\sqrt{2}} i \frac{\not-\not+\check{m}_{b}}{(k-q)^{2}-\check{m}_{b}^{2}} \\
& \frac{i}{(p-k)^{2}-M_{Z}^{2}} \frac{f_{b i} \gamma_{5}}{\sqrt{2}} \frac{\mathrm{d}^{4} k}{(2 \pi)^{4}} \\
& +\sum_{j \ell k b} i \int \frac{f_{a j} \gamma_{5}}{\sqrt{2}} U_{j \ell} i \frac{\not+\hat{m}_{\ell}}{k^{2}-\hat{m}_{\ell}^{2}} U_{\ell k}^{*}\left(-\epsilon_{k b}\right) \frac{-h_{b} \gamma_{5}}{\sqrt{2}} \\
& i \frac{\not k-\not+\check{m}_{b}}{(k-q)^{2}-\check{m}_{b}^{2}} \frac{i}{(p-k)^{2}-M_{Z}^{2}} \frac{f_{b i} \gamma_{5}}{\sqrt{2}} \frac{\mathrm{d}^{4} k}{(2 \pi)^{4}} .
\end{aligned}
$$

One sees immediately that the sum vanishes (trivially when the light neutrino masses $m_{\ell}$ are ignored, at order $m_{\ell}$ more accuracy is required). Respectively vanishes the contribution by the massive higgses. This is expected, since this coupling does not break the lepton number symmetry. It can be seen clearly also in the perturbative picture: the $\sigma$ lines appear always pairwise, one coming in and another going out.

Due to the scalar mixing there will arise also new type of graphs as those shown in figure 1. The new contribution to the Dirac mass is

$$
\delta m_{a i}=\frac{h_{a} \check{m}_{a} f_{a i}}{32 \pi^{2}} \sin 2 \theta\left(M_{1}^{2}-M_{2}^{2}\right) I\left(\check{m}_{a}, M_{1}, M_{2}\right) .
$$

There are two sets of graphs contributing to the majoron coupling. Those with the majoron in the fermion leg read

$$
\delta \xi_{a i}^{(a)}=\frac{h_{a} h_{a} f_{a i}}{64 \sqrt{2} \pi^{2}} \sin 2 \theta\left(M_{2}^{2}-M_{1}^{2}\right) I\left(\check{m}_{a}, M_{1}, M_{2}\right) .
$$

Another set consists of graphs where the majoron touches the scalar loop. The effective cubic couplings of a massive scalar to two majorons $\left(D_{i} H_{i} J J\right)$, can be written explicitly in the mass basis of the neutral scalars as

$$
\begin{aligned}
D_{J 1} & =-\frac{h_{a}}{\sqrt{2} \check{m}_{a}} M_{1}^{2} \sin \theta \\
D_{J 2} & =\frac{h_{a}}{\sqrt{2} \check{m}_{a}} M_{2}^{2} \cos \theta
\end{aligned}
$$

where $a$ is arbitrary. Hence one obtains

$$
\begin{aligned}
\xi_{a i}= & \frac{h_{a} f_{a i} h_{a}}{2 \sqrt{2}} \sin \theta \cos \theta \frac{1}{16 \pi^{2}} \\
& {\left[M_{1}^{2} I\left(\check{m}_{a}, 0, M_{1}\right)-M_{2}^{2} I\left(\check{m}_{a}, 0, M_{2}\right)\right] }
\end{aligned}
$$


Now, using the identity

$$
\begin{aligned}
& \left(M_{2}^{2}-M_{1}^{2}\right) I\left(\check{m}_{a}, M_{1}, M_{2}\right)= \\
& M_{2}^{2} I\left(\check{m}_{a}, 0, M_{2}\right)-M_{1}^{2} I\left(\check{m}_{a}, 0, M_{1}\right)
\end{aligned}
$$

one sees that these loops cancel, so that the result is zero. Hence we find that the mixing of the scalars does not induce any left-right components for the majoron coupling matrix. 


\section{REFERENCES}

[1] D. Dicus et al., Ap. J. 221, 327 (1978).

[2] P. B. Pal, Nucl. Phys. B 227, 237 (1983).

[3] G. Steigman and M. S. Turner, Nucl. Phys. B 253, 375 (1985), and refs. therein.

[4] M. Gell-Mann, P. Ramond, and R. Slansky, in Supergravity, edited by P. van Nieuwenhuizen and D. Z. Freedman (North Holland, Amsterdam, 1979).

[5] T. Yanagida, in Proc. Workshop on the Unified Theory and the Baryon Number of the Universe, edited by O. Sawada and S. Sugamoto (KEK, Tsukuba, 1979).

[6] Y. Chikashige, R. N. Mohapatra, and R. Peccei, Phys. Lett. 98B, 265 (1981).

[7] J. Schechter and J. W. F. Valle, Phys. Rev. D 25, 2951 (1982).

[8] H. Georgi, S. Glashow, and S. Nussinov, Nucl. Phys. B 193, 297 (1981).

[9] P. B. Pal, in Proc. Beyond the Standard Model II, Norman, OK, U.S.A., edited by K. A. Milton, R. Kantowski, and M. Samuel (World Scientific, Singapore, 1990), p. 373.

[10] J. Cline, K. Kainulainen, and S. Paban, Phys. Lett. B 319, 513 (1993).

[11] A. Zee, Phys. Lett. 93B, 389 (1980).

[12] A. Zee, Phys. Lett. 161B, 141 (1985).

[13] S. Bertolini and A. Santamaria, Nucl. Phys. B 310, 714 (1988).

[14] H. Kikuchi and E. Ma, Phys. Lett. B 335, 444 (1994).

[15] Y. Chikashige, R. N. Mohapatra, and R. Peccei, Phys. Rev. Lett. 45, 1926 (1980).

[16] R. N. Mohapatra and P. B. Pal, Phys. Rev. D 38, 2226 (1988).

[17] K. Kanaya, Prog. Theor. Phys. 64, 2278 (1980).

[18] Y. B. Zeldovich, Akad. Nauk. SSSR 86, 505 (1952).

[19] E. J. Konopinski and H. M. Mahmoud, Phys. Rev. 92, 1045 (1953).

[20] S. Petcov, Phys. Lett. 110 B, 245 (1982).

[21] J. W. F. Valle, Phys. Lett. 159B, 49 (1985).

[22] J. T. Peltoniemi, Mod. Phys. Lett. A 38, 3593 (1993).

[23] K. S. Babu, Phys. Lett. 203B, 132 (1988).

[24] G. Gelmini and M. Roncadelli, Phys. Lett. 99B, 411 (1981).

[25] J. T. Peltoniemi and J. W. F. Valle, Phys. Lett. B 304, 147 (1993).

[26] G. Gelmini, S. Nussinov, and M. Roncadelli, Nucl. Phys. B 209, 157 (1982). 


\section{FIGURES}

FIG. 1. The diagrams generating the mass correction to the left-handed states in the singlet majoron model.

FIG. 2. The diagrams generating the corrections to the majoron coupling with the left-handed neutrino states in the singlet majoron model.

FIG. 3. The diagrams contributing to the Dirac mass term in the singlet majoron model

FIG. 4. The would-be diagrams contributing to the majoron coupling between left- and right-handed states. These graphs cancel.

FIG. 5. The diagrams giving the correction to the mass term for the right-handed states in the singlet majoron model.

FIG. 6. The diagrams generating the lowest order corrections to the majoron coupling between the right-handed states in the singlet majoron model.

FIG. 7. One loop diagrams contributing to the neutrino mass and to the neutrino-majoron coupling in the triplet majoron model.

FIG. 8. The most important one-loop diagrams contributing to the neutrino mass and the neutrino-majoron coupling in the doublet majoron model.

FIG. 9. The diagrams generating the mass and the majoron coupling in the Zee model with singlet majoron.

FIG. 10. The diagrams generating the mass and the majoron coupling in the Babu model with singlet majoron.

FIG. 11. Diagrams apparently contributing to the coupling of the majoron to the charged

leptons, in a model where a singlet majoron couples directly only to scalars. These graphs cancel. 
(a)

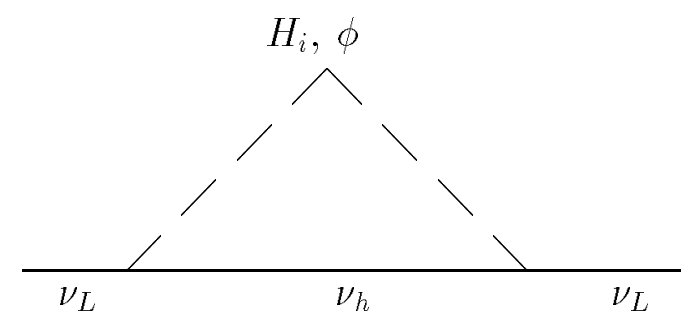

(b)

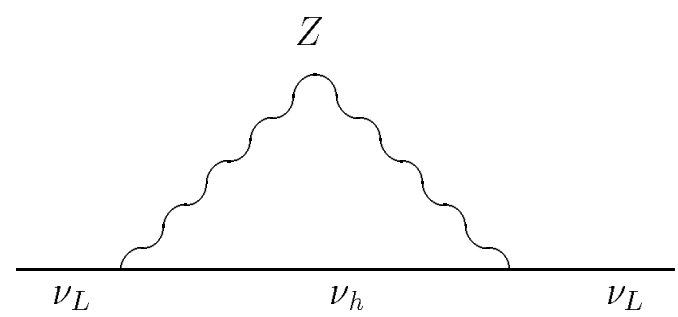

figure 1 
(a)

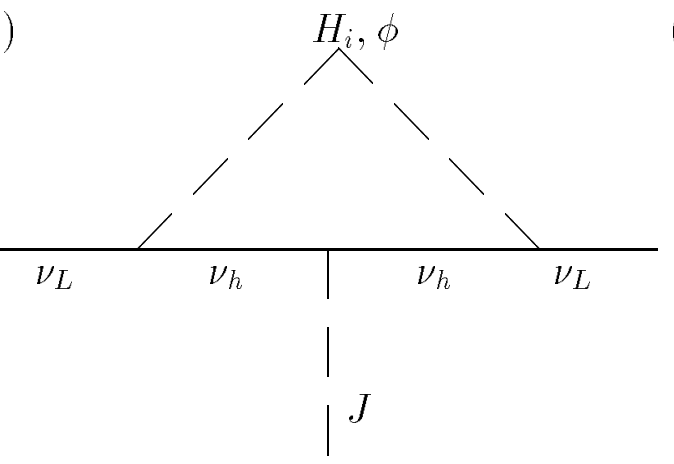

(b)

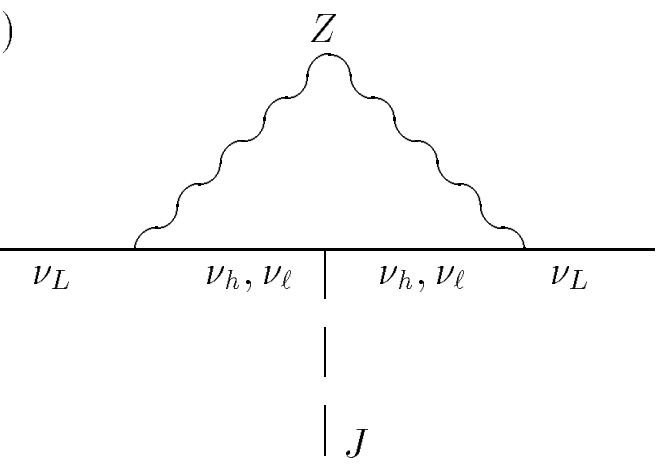

Figure 2 


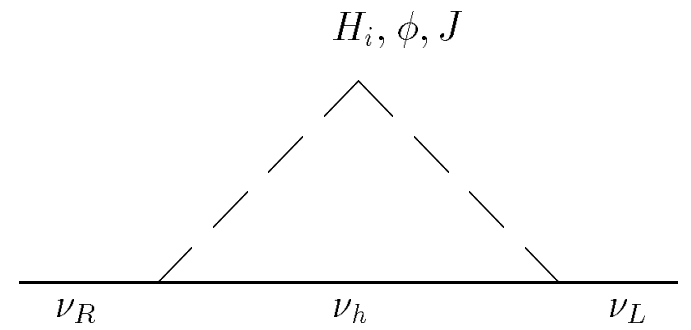

Figure 3 
(a) $\quad \mid$

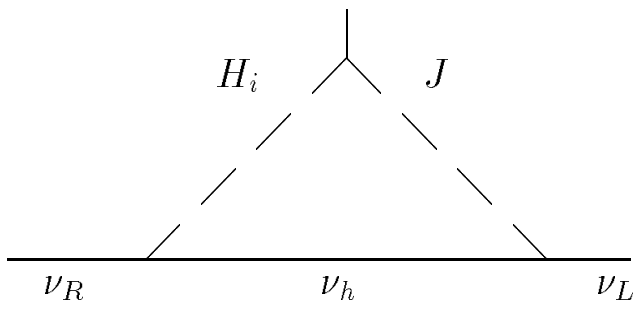

(c)

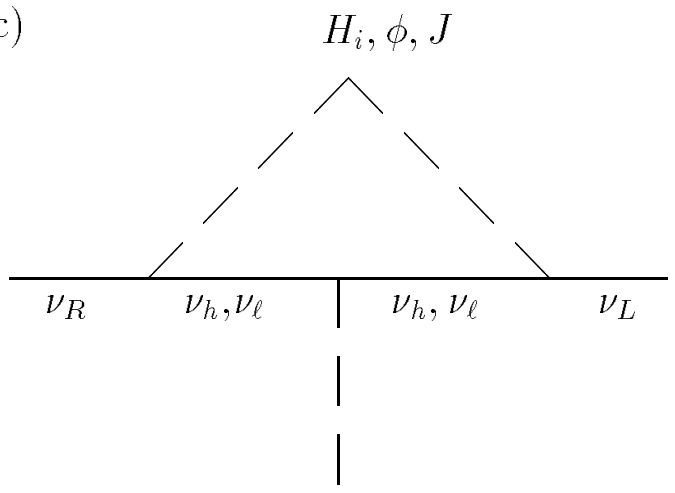

(b)

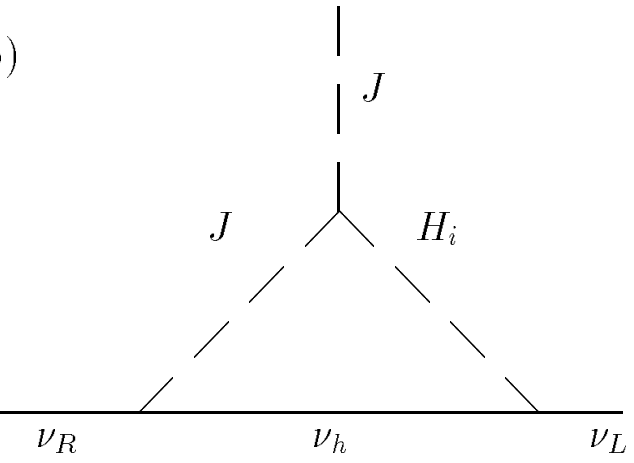

Figure 4 


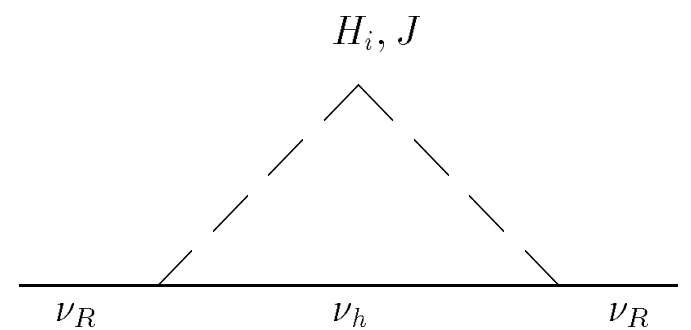

Figure 5 
(a)

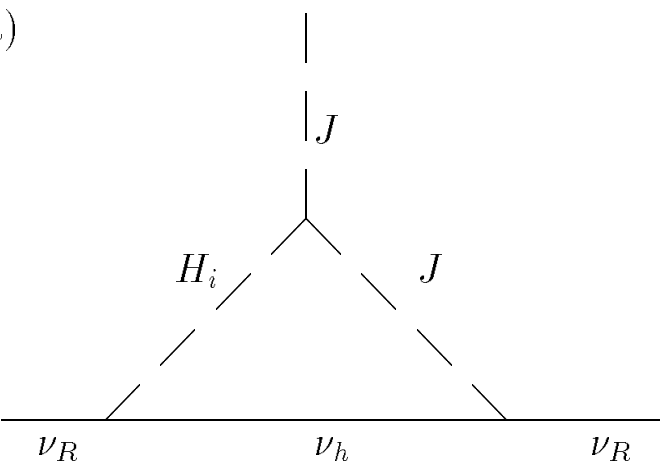

$H_{i}, J$

(b)

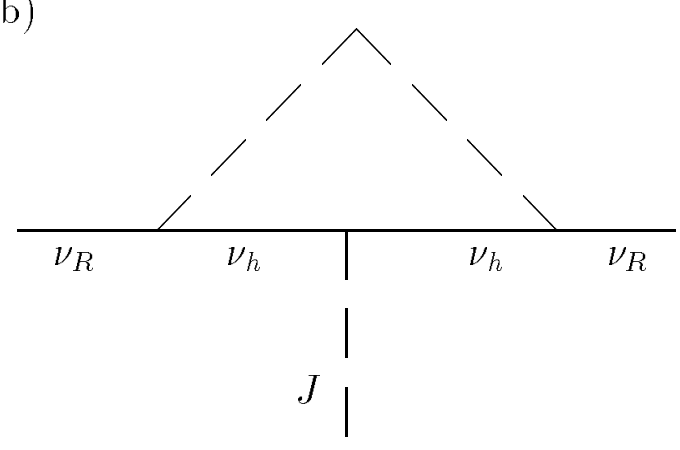

Figure 6 

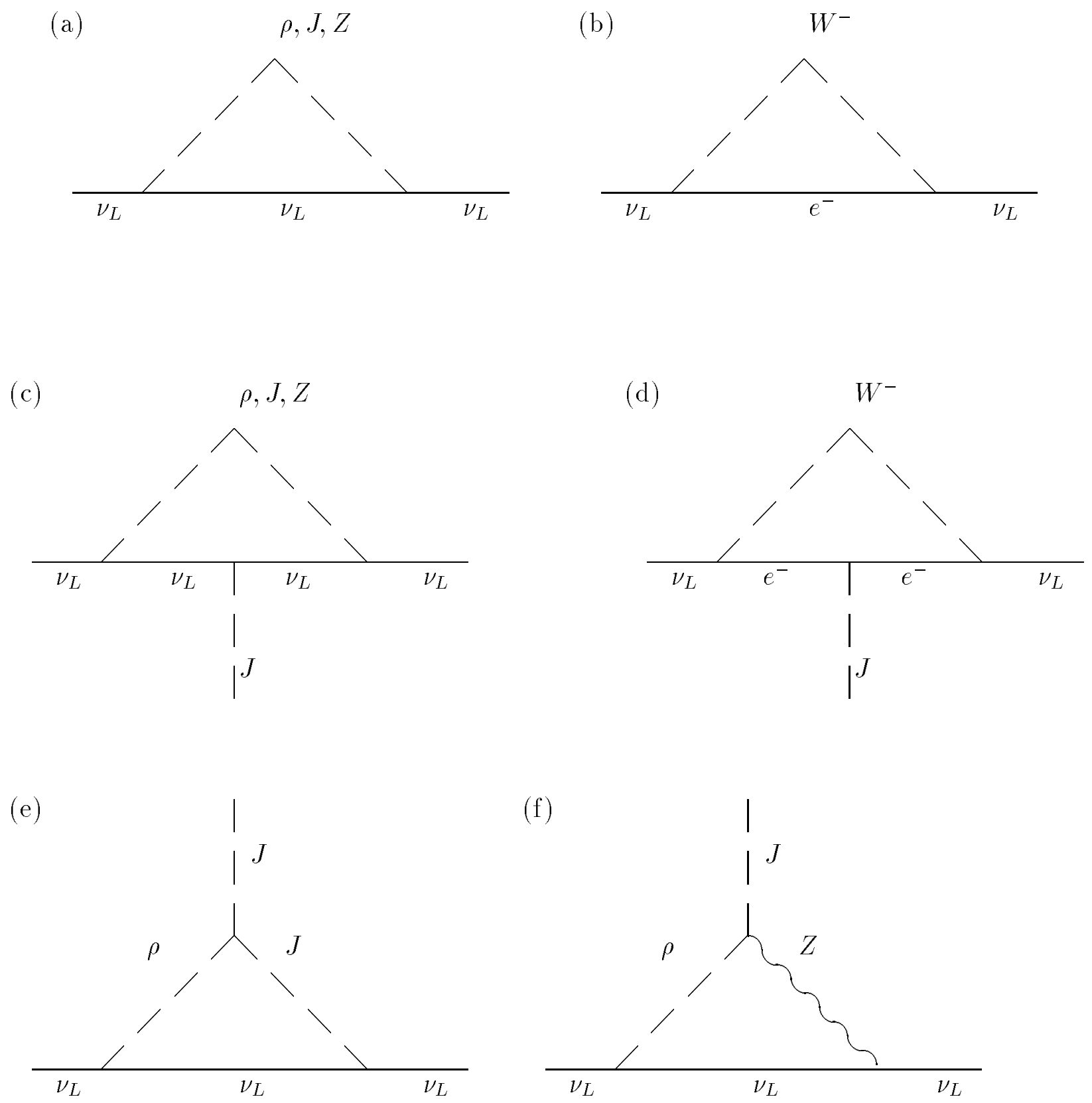

Figure 7 

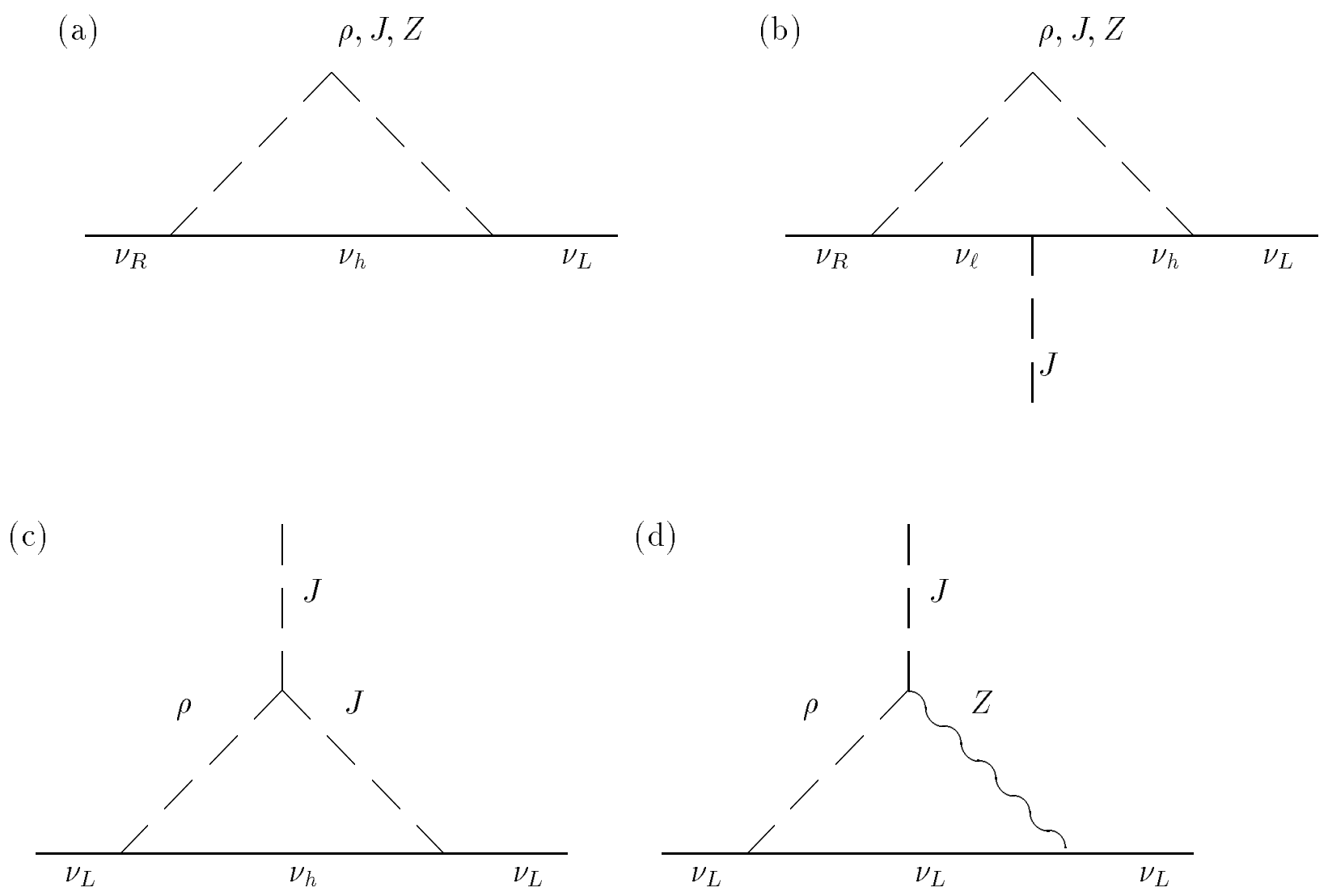

Figure 8 
(a)

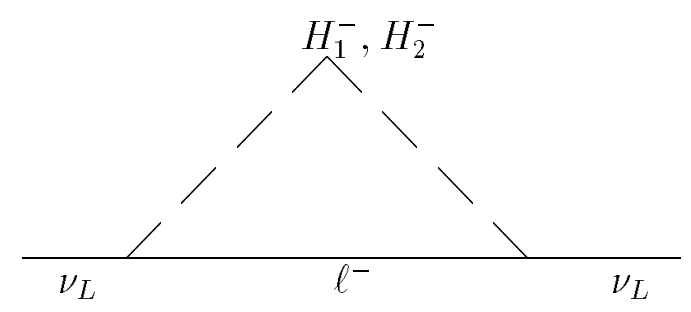

(b) $\quad \mid$

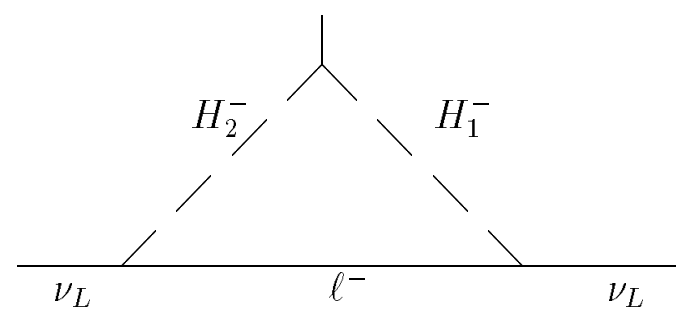

Figure 9 
(a)

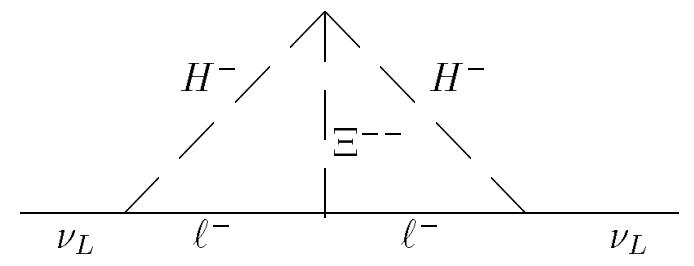

(b) $\quad \mid$

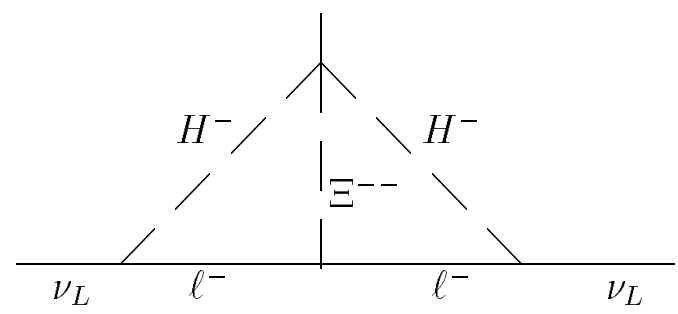

Figure 10 
(b) $\left.\quad\right|^{J}$

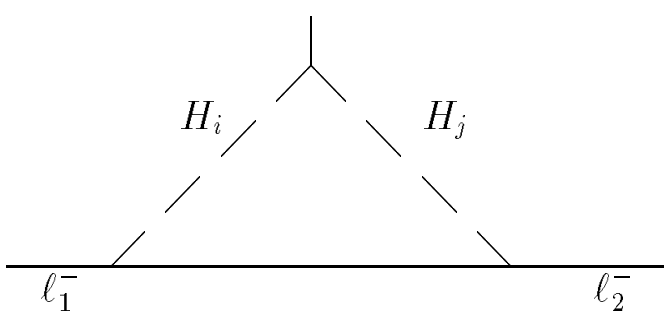

Figure 11 